\title{
Management of a female with recurrence of fibromatosis of the chest wall adjacent to the breast: a case report
}

\author{
Cheng Shen, Yubin Zhou and Guowei Che*
}

\begin{abstract}
Extra-abdominal desmoid tumor is a rare soft tissue tumor that is histologically benign, but may behave aggressively. This case report specifically describes the clinical, radiographic, and pathologic features of 27 year-old female who experienced a post-surgical recurrence of fibromatosis of the chest wall over a two-year period of time secondary to previous inadequate excision. The fibromatosis was found to be involving the lower-inner quadrant of her right breast and causing worsening pain. A surgical management strategy was successfully undertaken.
\end{abstract}

Keywords: Aggressive fibromatosis, Recurrent desmoid tumour, Chest wall, Breast

\section{Background}

Desmoid tumor is an aggressive fibromatosis that may occur in abdominal and extra-abdominal areas [1]. They constitute less than $0.03 \%$ of all neoplasms [2]. For extra-abdominal desmoids, local recurrence rates range from $24 \%$ to $77 \%$ in reported series [3-5]. Although the fibromatosis of chest wall represents $8-10 \%$ of all cases and surgery is the primary treatment modality, there remains a significant lack of agreement amongst surgeons on how to manage the disease that is suspected to have accompanied involvement of adjacent breast especially for an unmarried woman. We report a case where the tumour had a repeated recurrence over a 2 -year period.

\section{Case presentation}

A 27 year-old unmarried female presented to our hospital with worsening pain of the right chest wall region and a recurrent obvious mass adjacent to the lowerinner quadrant of her right breast for 5 months. Two years prior to her current presentation, she presented to a local hospital with a palpable right chest wall mass close to the same quadrant of right breast and underwent a surgical resection. The histological examination showed a typical picture of an aggressive fibromatosis.

\footnotetext{
* Correspondence: cheguowei@yahoo.com.cn

Department of Thoracic Surgery, West-China Hospital, Sichuan University, Chengdu 610041, China
}

Physical examination showed a $15 \mathrm{~cm}$ and well-healed surgical scar along the inferior-inner aspect of her right inframammary fold. Plain and contrast-enhanced chest and epigastrium computed tomography (CT) showed a $10 \times 5 \mathrm{~cm}$ mass on the right inferior chest wall that appeared to be in continuity with the right pectoralis major muscle and right rectus abdominis muscle (Figure 1A). Magnetic Resonance Imaging (MRI) of the breast and the chest confirmed a $9 \times 4 \mathrm{~cm}$ mass in close proximity to the lower-inner quadrant of the right breast, with contact to the ribs (Figure 1B).

As diagnosis was established, surgery was scheduled. Under general anesthesia with selective intubation, the patient lay on the operating table with supine position. She underwent an en bloc resection of the tumor and the underlying musculature (inferior lateral portion of the right pectoralis major muscle and superior portion of the right rectus abdominis musculature, and anterior portion of the right latissimus dorsi muscle) and en bloc resection of the underlying chest wall structures (seventh rib and intercostal muscle). The defect on the right chest wall was then closed with a $10 \times 10 \mathrm{~cm}$ Dacron patch. Although the mass was in close proximity to the lowerinner quadrant of the right breast, all surgical margins were negative with the intraoperative frozen section and the right mastectomy was avoided. Then the right breast was closed in the skin flap plasty. Patient was transferred to the intensive care unit and moved to general care 

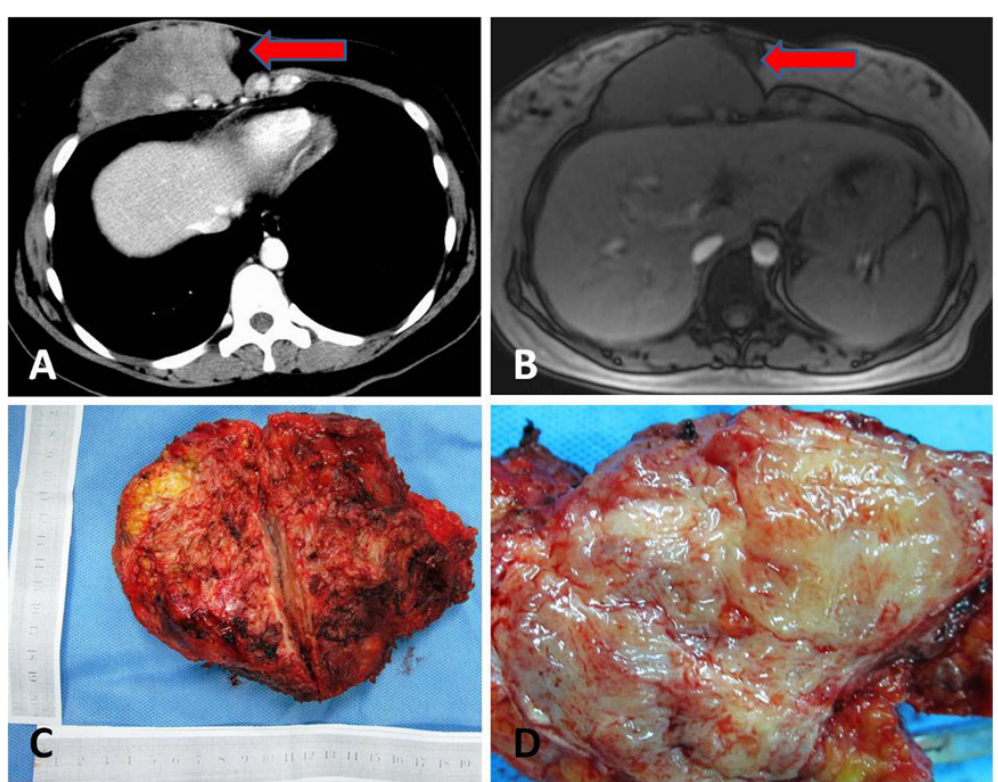

Figure $1 \mathrm{CT}$ and MRI showed a mass on the right inferior chest wall and the general view of the specimen. A: Computed tomography showed a $10 \times 5 \mathrm{~cm}$ mass on the right inferior chest wall that appeared to be in continuity with the right pectoralis major muscle and right rectus abdominis muscle. B: Magnetic Resonance Imaging of the breast and the chest confirmed a $9 \times 4 \mathrm{~cm}$ mass in close proximity to the lower-inner quadrant of the right breast, with contact to the ribs. C: The surgically removed specimen was lobulated and $14 \times 16 \times 10 \mathrm{~cm}$ in size, containing a 10x12×8 cm tumor. D: Bisected tumor showed a grossly circumscribed tumor with white, whorled patterns and without necrosis.

after 24 hours and she was discharged to home after a week. She is currently under follow-up.

The surgically removed specimen was lobulated and $14 \times 16 \times 10 \mathrm{~cm}$ in size, containing a $10 \times 12 \times 8 \mathrm{~cm}$ tumor. Macroscopically, the bisected tumor showed a grossly circumscribed firm tumor with white, whorled patterns and without necrosis (Figure 1C, D). Microscopically, the lesion was composed of evenly spaced plump spindle cells arranged in intersecting fascicles and associated with mild to moderate amounts of collagen resembling
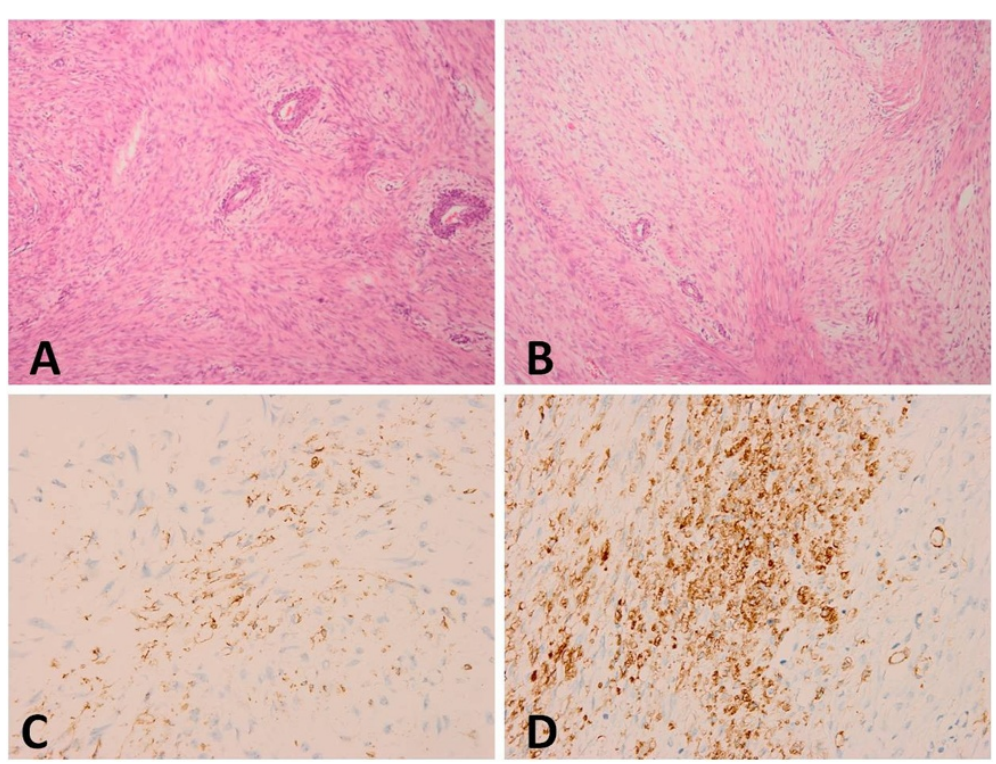

Figure $\mathbf{2}$ Histological features. Section stained with hematoxylin and eosin reveals evenly spaced plump spindle cells arranged in intersecting fascicles and associated with mild to moderate amounts of collagen resembling keloid (A, B original magnification $\times 100)$. On immunohistochemical staining, the spindle cells were positive for muscle-specific actin $(M S A)(\mathbf{C}$, original magnification $\times 400)$ and smooth muscle actin $($ SMA) $(\mathbf{D}$, original magnification $\times 400)$. 
keloid (Figure 2A, B). On immunohistochemical staining, the spindle cells were negative for S-100 protein and Epithelial Membrane Antigen (EMA). The spindle cells were positive for muscle-specific actin (MSA) (Figure 2C) and smooth muscle actin (SMA) (Figure 2D). The histology and immunohistochemical staining supported a diagnosis of fibromatosis (desmoid tumor).

\section{Conclusions}

Extra-abdominal desmoids are frequently referred to as fibromatoses that are mesenchymal neoplasms and develop from the connective tissues, fasciae and aponeuroses.

For the treatment of this recurrent tumour, an aggressive surgical resection strategy with a safe margin (2-3 $\mathrm{cm})$ remains the standard therapeutic anoeuvre, which has been strongly advocated by surgeons dealing with fibromatosis of the chest wall that involves the breast $[6,7]$. The reasons that support this aggressive surgical approach include the potentials of fibromatosis to grow aggressively locally and to invade into the surrounding structures, thus having a high rate of local recurrence when incompletely excised with positive surgical margins. Some surgeons, however, disagree with such a wide excision and prefer more conservative resection [8-10]. Their concerns are about the less optimal cosmetic outcome and the risk of loss of function. Further, there have not been any reports showing fibromatosis can metastasize. Presence of residual tumor cannot be clearly shown to impact disadvantageously on five-year disease free or overall survival [3]. In our case, we chose to perform a wide en bloc resection to avoid future recurrence, based on negative frozen section on the surgical margin. Thus, we were able to avoid mastectomy. A flap plasty and cosmetology of breast were performed. This is particularly beneficial to the young woman who was going to get married in the next year and her quality of life was not adversely affected.

For a large recurrent fibromatosis involving the chest wall and breast, wide en bloc resection is necessary to prevent future recurrence but shall avoid mastectomy if possible. This surgical management is particularly useful for unmarried young women.

\section{Consent}

Written informed consent was obtained from the patient for publication of this case report and any accompanying images. A copy of the written consent is available for review by the Editor-in-Chief of this journal.

\section{Competing interests}

The authors declare that they have no competing interests.

\section{Authors' contributions}

CS was involved in drafting the manuscript. YZ was involved in acquisition of data and preparing the figures. GC designed and revised the manuscript. All authors have read and approved the final manuscript.

\section{Acknowledgments}

We greatly appreciate the assistance of the staff of the Department of Thoracic Surgery, West-China Hospital, Sichuan University, and thank them for their efforts.

Received: 19 September 2012 Accepted: 4 March 2013

Published: 8 March 2013

References

1. Enzinger FM, Shiraki M: Musculo-aponeurotic fibromatosis of the shoulder girdle (extra-abdominal desmoid). Analysis of thirty cases followed up for ten or more years. Cancer 1967, 20:1131-1140.

2. McKinnon JG, Neifeld JP, Kay S, et al: Management of desmoid tumors. Surg Gynecol Obstet 1989, 169:104-6.

3. Merchant NB, Lewis JJ, Woodruff JM, Leung DH, Brennan MF: Extremity and trunk desmoid tumors: a multifactorial analysis of outcome. Cancer 1999, 86:2045-52.

4. Allen PJ, Shriver CD: Desmoid tumors of the chest wall. Semin Thorac Cardiovasc Surg 1999, 11:264-9.

5. Ferenc T, Sygut J, Kopczynski J, et al: Aggressive fibromatosis (desmoid tumors): definition, occurrence, pathology, diagnostic problems, clinical behavior, genetic background. Pol J Pathol 2006, 57:5-15.

6. Thomas T, Lorino C, Ferrara JJ: Fibromatosis of the breast: a case report and literature review. J Surg Oncol 1987, 35:70-74.

7. Abbas AE, Deschamps C, Cassivi SD, Nichols FC, Allen MS, Schleck CD, Pairolero PC: Chest wall desmoid tumors: Results of surgical intervention. Ann Thorac Surg 2004, 78:1219-1223.

8. Godwin Y, McCulloch TA, Sully L: Extra-abdominal desmoid tumour of the breast: review of the primary management and implications for breast reconstruction. Br J Plast Surg 2001, 54:268-271.

9. Matherne TH, Green A, Tucker JA, Dyess DL: Fibromatosis: the breast cancer imitator. South Med J 2004, 97:1100-1103.

10. Schwarz GS, Drotman M, Rosenblatt R, Milner L, Shamonki J, Osborne MP: Fibromatosis of the breast: case report and current concepts in the management of an uncommon lesion. Breast J 2006, 12:66-71.

doi:10.1186/1749-8090-8-41

Cite this article as: Shen et al: Management of a female with recurrence of fibromatosis of the chest wall adjacent to the breast: a case report. Journal of Cardiothoracic Surgery 2013 8:41.

\section{Submit your next manuscript to BioMed Central and take full advantage of:}

- Convenient online submission

- Thorough peer review

- No space constraints or color figure charges

- Immediate publication on acceptance

- Inclusion in PubMed, CAS, Scopus and Google Scholar

- Research which is freely available for redistribution 